\title{
Research on lubrication performance of micro-textured journal bearing based on fluent
}

\author{
Lili Wang ${ }^{a}$, Shaohui Guo ${ }^{b}$, Guoxiao Yin ${ }^{c}$ \\ Shandong University of Science and Technology, Qingdao 266590, China. \\ awang-ly-ly@163.com, bm17806258200@163.com, c598925940@qq.com
}

\begin{abstract}
The micro-textured surface technology has obvious effect on improving the lubrication performance of the journal bearing. In this paper, a 3D micro-textured journal bearing model is established by fluent and compared with non-textured smooth journal bearing. The effect of circumferential total texture and partial texture of different positions in bearing area on bearing lubrication performance is analyzed. Results show that circumferential total texture and partial texture can effectively improve the bearing capacity of bearing and reduce the friction coefficient, and there is a reasonable and effective range for the quantity, position and density of micro texture.
\end{abstract}

Keywords: Micro-texture; journal bearing; bearing capacity; friction coefficient.

\section{Introduction}

Because of the good performances such as large carrying capacity, stable operation and high operating accuracy, journal bearing is widely used in the engineering fields such as steam turbine, railway locomotive, high precision lathe and aerospace. It has great significance for extending the service life of the sliding bearing and improving the stability of the mechanical system to study the lubrication characteristics of the sliding bearing, and improve continuously the working performance of the bearing. The micro-textured surface technology has obvious effect on improving the bearing capacity of the journal bearing, reducing the friction coefficient and forming the oil film. Many scholars have done a lot of research on the application of micro-textured surface technology in journal bearing.

Hamilton et al. [1] studied the theory and experiment of micro texture through processing a series of micro bumps on the mechanical seal by the etching technique, proposed that dynamic pressure effect is obtained by micro convex on the surface of friction pairs. The influence of different texture shapes including rectangles, circles, triangles on working performance of journal bearing was researched based on Reynolds equation by Rahmani et al. [2]. Brajdic-Mitidieri et al. [3] used the CFD method to carry on the numerical simulation for the infinite long slide bearing which only had one rectangle texture. Yang [4] analyzed the effect of different geometric parameters and sectional shapes of micro texture on the lubrication performance of journal bearing by numerical analysis method. Qi [5] established an infinite long radial bearing lubrication model, and researched the influences of surface texture quantity and sizes on bearing performance by CFD method based on N-S equation. Zhao et al. [6] researched the effect of partial groove texture on the lubrication performance of bearing using CFD model. In consideration of the cavitation and turbulence effect, Yin et al. [7] studied the influence of geometrical parameters and distribution characteristics of surface micro texture on the bearing performance of journal bearing. Qiu [8] built the simulation model by Fluent, and studied the relationship between the shape of the cross section and the carrying capacity, the effect of the texture ratio and speed on the bearing capacity.

This paper builds the 3D micro-textured journal bearing model by fluent based on N-S equation, the effect of quantity, position and density of surface micro-texture on bearing lubrication performance is analyzed. The research results enrich the understanding of the effect of micro texture technology on the performance of journal bearings, and there is great significance for the application of surface texture technology in journal bearing. 


\section{Model}

In this paper, the model of hydrodynamic sliding bearing is studied. As shown in Figure 1, radius of bearing bush $R_{1}$ is $20 \mathrm{~mm}$, diameter of axle $R_{2}$ is $19.9 \mathrm{~mm}$, initial deflection angle $\psi$ is $40^{\circ}$, eccentricity $e$ is $0.06 \mathrm{~mm}, \theta_{\mathrm{i}}$ is the starting angle of circumferential coverage angle, $\Delta \theta$ is the circumferential coverage angle. The lubricating condition of the sliding bearing is incompressible, constant temperature and laminar flow state, lubricating method is oil lubrication, the parameters of lubricating oil is as following: density $p$ is $785 \mathrm{~kg} / \mathrm{m}^{3}$, dynamic viscosity $\eta$ is $0.035 \mathrm{~Pa}$.s. As shown in Fig. 2, the paper adopts the groove micro texture with cross section shape of rectangular, the length of texture unit $L$ is $20 \mathrm{~mm}$, height $D$ is $0.05 \mathrm{~mm}$, width $W$ is $0.3 \mathrm{~mm} . N$ represents the quantity of micro texture.

The setting of boundary conditions is as following: pressure exit boundary conditions are used, two end of the oil film is set to the pressure outlet, stress is the atmospheric pressure by default, the inner surface of the bearing bush is set to a fixed wall, the surface of axle is set to a revolving wall, and rotation rate is $n=600 \mathrm{rad} / \mathrm{s}$, the direction of rotate is clockwise, the region between the two surfaces is the calculated fluid region.

The pressure distribution of oil film can be obtained by analyzing the finite element model of micro-texturing journal bearing using fluent, then the bearing capacity, friction force and friction coefficient of the bearing in the corresponding state can be solved.

The calculation of bearing capacity

The bearing capacity can be obtained by integrating the oil film pressure:

$\mathrm{F}_{\mathrm{y}}=\iint p \cdot \mathrm{d} x \cdot d y$
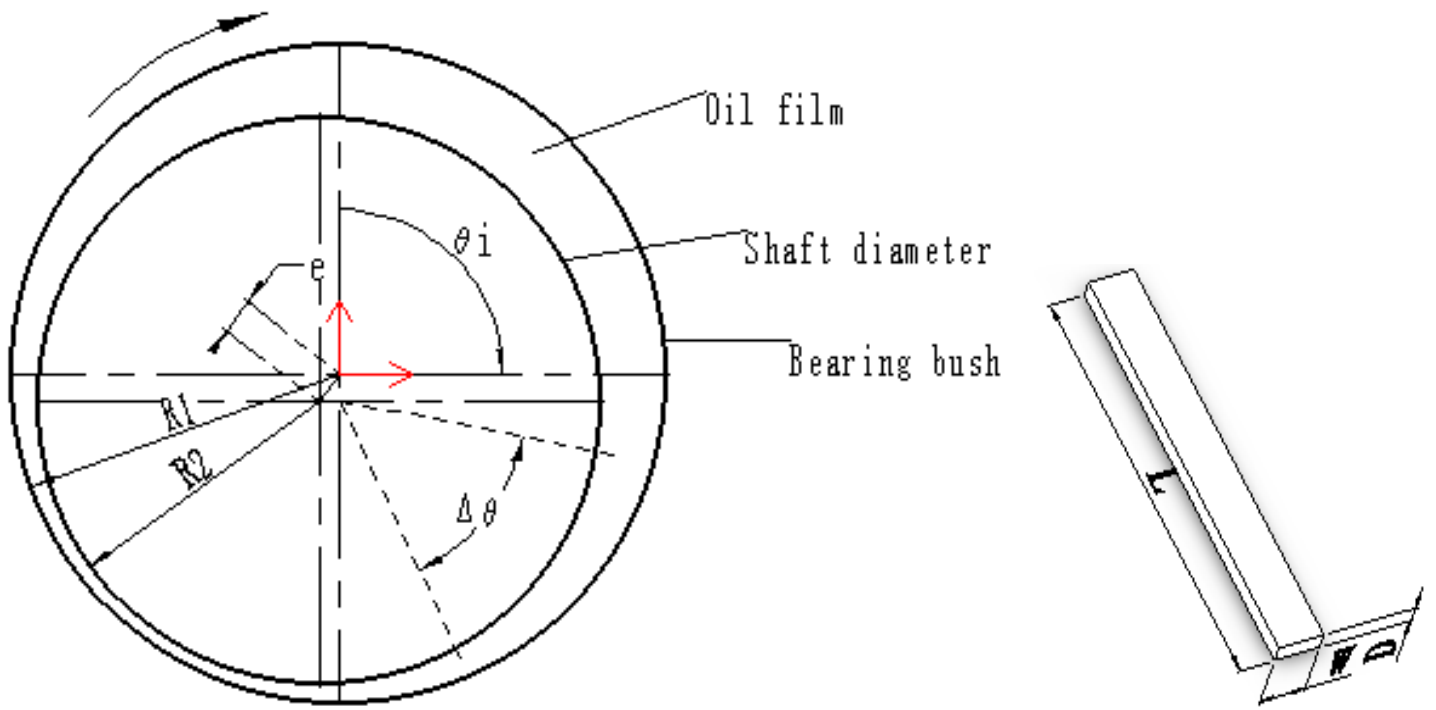

Fig.1 Diagram of journal bearing

Fig.2 Diagram of micro-textured unit

The calculation of friction force

The friction force can be obtained by integrating the viscous shear stress acting in the journal:

$\mathrm{F}_{f}=\iint \tau \cdot d x \cdot d y$

The calculation of friction coefficient

The friction coefficient can be obtained by the ratio of friction force and bearing capacity:

$f=\frac{F_{f}}{F_{\mathrm{y}}}=\frac{\iint \tau \cdot d x \cdot d y}{\iint p \cdot d x \cdot d y}$ 


\section{Results and analysis}

\subsection{The circumferential total texture on journal bearing}

The circumferential total texture of journal bearing is set to rectangular texture in circumferential position uniformly, $\theta_{i}=0^{\circ}, \Delta \theta=360^{\circ}$, the texture number $N$ is set to $0,6,8,10,15,18,20$, and $N=0$ represents smooth bearing without texture .

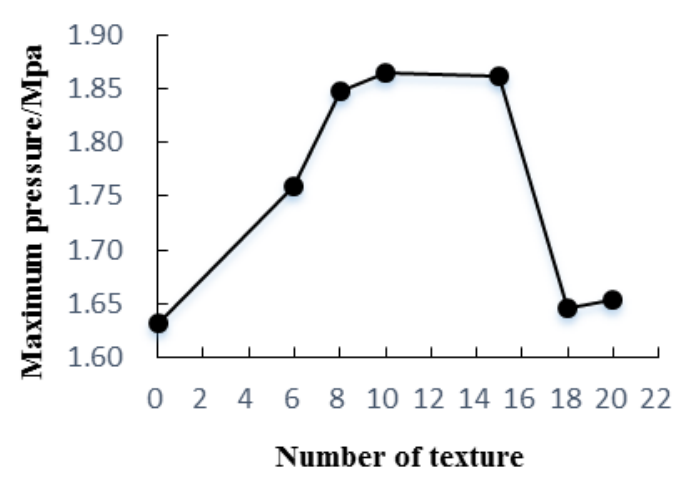

(a) Maximum pressure of oil film

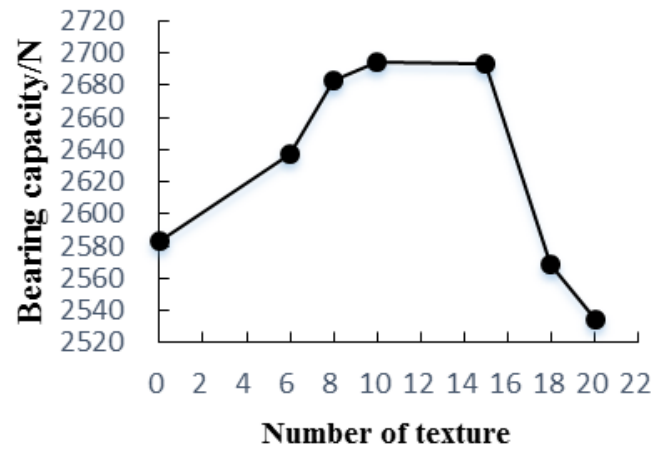

(b) Bearing capacity

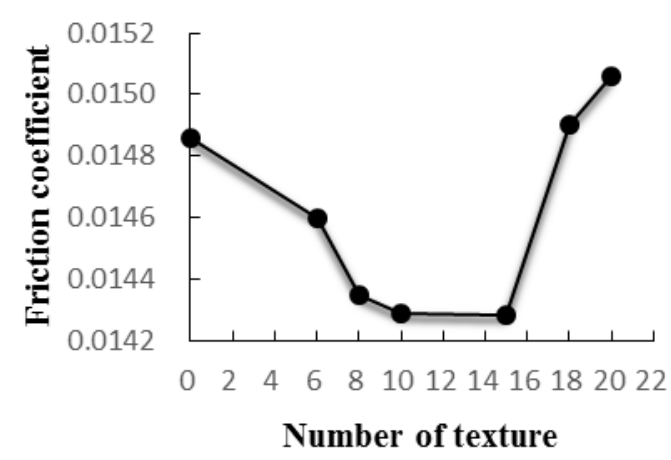

(c) Friction coefficient

Fig. 3 The lubrication performance of bearing with different number textures

The (a), (b), (c) of Fig. 3 represents maximum pressure of oil film, bearing capacity, and friction coefficient respectively when the circumferential total textures of journal bearing has different numbers. As shown in Fig. 3, compared with the smooth bearing, both the maximum pressure and bearing capacity can be improved through processing circumferential total texture, the friction coefficient obviously reduces by this method, the lubrication performance of bearing is improved significantly. This is because that micro texture is conducive to producing dynamic pressure lubrication when circumferential total textures of journal bearing has different numbers, and micro texture can store lubricating oil and reduce friction force. With the increase of the number of texture, the lubrication performance of bearing reaches the best; but when the number of texture exceeds 18 , the bearing capacity and maximum pressure of journal bearing with circumferential total texture are lower than the smooth bearing without texture, and the friction coefficient is greater than smooth bearing. Results show that the lubrication performance of bearing including bearing capacity, maximum pressure and the friction coefficient can be improved when bearing has proper amount and distribution density of circumferential total texture; but there is a reasonable and effective range for the distribution density of micro texture, it can produce adverse effect on performance of bearing when density of micro texture exceeds a certain range.

\subsection{The partial texture on journal bearing}

In consideration of the limitations of the texture processing technology and the actual engineering application of journal bearing, the partial texture not only saves the processing cost but also reduces the difficulty of processing texture. So it has an important significance for engineering application to research the influence of the partial texture on journal bearing. As shown in Tab.1, this paper choices three positions in the bearing zone as studying object (position 0 indicates smooth bearing), micro 
texture which covers a circumferential area of $1 / 2,1 / 4,1 / 12$ is processed respectively. $\theta_{i}$ is the starting angle of circumferential coverage angle , $\Delta \theta$ is the circumferential coverage angle, $N$ is the quantity of micro texture.

Table 1 The different positions of the partial texture

\begin{tabular}{rcccc}
\hline $\begin{array}{c}\text { Different } \\
\text { positions }\end{array}$ & $\theta \mathrm{i}$ & $\Delta \theta$ & $\begin{array}{c}\text { Number } \\
N\end{array}$ & $\begin{array}{c}\text { Maximum pressure } \\
/ \mathrm{MPa}\end{array}$ \\
\hline position 0 & $0^{\circ}$ & $0^{\circ}$ & 0 & 1.632 \\
\hline position 1 & $0^{\circ}$ & $180^{\circ}$ & 10 & 1.713 \\
\hline position 2 & $90^{\circ}$ & $90^{\circ}$ & 10 & 1.873 \\
\hline position 3 & $150^{\circ}$ & $30^{\circ}$ & 20 & 1.953 \\
\hline
\end{tabular}

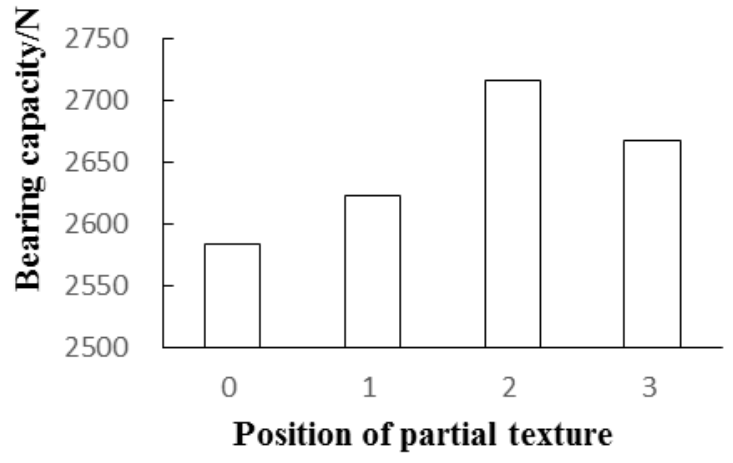

(a) Bearing capacity

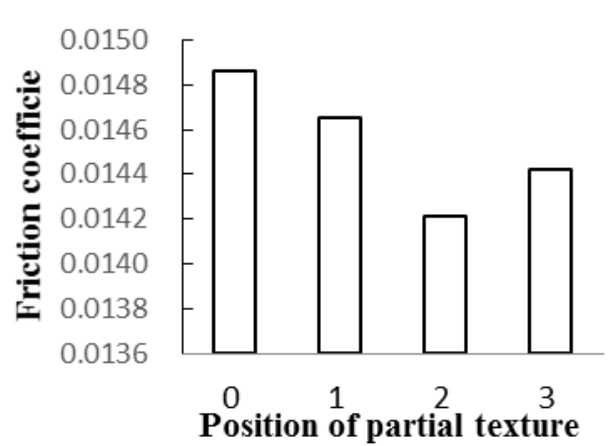

(b) Friction coefficient

Fig.4 The lubrication performance of bearing with different position textures

The fig.(a)and (b) of Fig. 4 represents bearing capacity and friction coefficient respectively when the partial textures of journal bearing has different numbers. The Tab.1 shows that the maximum pressure in oil film of bearing with partial texture is bigger than smooth bearing without texture; as can be seen from Fig. 4(a) and (b) that the bearing capacity of three positions with partial texture is bigger than smooth bearing, and friction coefficient is smaller than smooth bearing, this illustrates the partial texture can improve the lubrication performance of journal bearing; from the numerical change trend by comparing with the three positions, the cover area of position 2 is less than position 1 , and the number of texture (the distribution density) is smaller than position 3 , but the bearing capacity of journal bearing is improved obviously and friction coefficient is the minimum. The above results show that the lubrication performance of journal bearing can be improved more effectively when the bearing area has partial texture in the reasonable position, and there is an optimal position and covering area for improving the bearing capacity. For a fixed size of texture, the improving of lubrication performance has a certain relationship with the quantity and density of micro texture, there is an optimal quantity and density of micro texture for improving the lubrication performance of journal bearing.

\section{Discussion \& Conclusions}

In this paper, to reveal the effects of surface texture on the performance of journal bearing, the effect of circumferential total texture and partial texture on bearing lubrication performance is analyzed through establishing a 3D micro-textured journal bearing model. The main conclusions are the following:

a) The circumferential total texture can improve the bearing capacity of journal bearing and the maximum pressure of oil film, reduce the friction coefficient effectively, but there is a reasonable and effective range for the quantity and distribution density of micro texture. It can produce adverse effects on performance of bearing when density of micro texture exceeds a certain range. 
b) The reasonable partial texture in bearing area of journal bearing can also improve the lubrication performance, and there is an optimal position and density of micro texture to increase the bearing capacity and reduce the friction coefficient.

The influence of surface texture on the journal bearing is influenced by many factors, so further research needs to consider various factors, and simulation research in this paper has some limitations. The theoretical analysis and experimental methods should be more used to analysis the micro texture technology based on simulation research, it has greater guiding significance for the practical application of micro texture technology in engineering.

\section{Acknowledgements}

This work is supported by National Natural Science Foundation of China (NO. 51305242) and supported by SDUST Research Fund (NO. 2015JQJH104)

\section{References}

[1] Hamilton D B, Halavit J A. A theory of lubrication by micro irregularities. Journal of Basic Engineering, 1966, 88(1):177-185.

[2] Rahmani R, Mirzaee I, Shirvani A, etal. An analytical approach for analysis and optimization of slider bearings with infinite width. Tribology International, 2010, 43:1551-1565.

[3] Brajdic-Mitidieri P, Gosman A D, Ioannides E, et al. CFD analysis of a low friction pocketed pad bearing. Journal of Tribology, 2005,127 (4):803-812.

[4] Yang Huarui. Study on the effects of surface texture and bubbly oil performance of journal bearings. Beijing: Beijing Institute of Technology, 2014.

[5] Qi Ye, Chang Qiuying, Li Huiting, Shen Zongze. Research on load carrying properties of surface textured journal bearing. Lubrication Engineering, 2012, 37(12):36-42

[6] Han Lei, Zhao Yuncai. CFD-analysis of partiall surface groove texture on hydrodynamic lubrication. China Surface Engineering, 2013, 26(06):112-118.

[7] Yin Minhu, Chen Guoding, Gao Dangcheng, Wang Lin. Effects of three types of surface texture on the performances of journal bearing. Journal of Harbin Institute of Technology, 2016, 48(1):159-164.

[8] Qiu Xiaocong, Chen Guoquan .CFD-analysis of groove texture on hydrodynamic lubrication. Mechanical and Electronic Technology, 2015, (1):48-59. 